\title{
Microfluidic Organ Chip for In Vitro Model of Blood Glucose Monitoring and Regulation
}

\author{
Yingqiang $\mathrm{Wu}^{1, \mathrm{a}}$, Guo $\mathrm{Wu}^{1, \mathrm{~b}}$, Pengcheng $\mathrm{Fu}^{2, \mathrm{c} *}$, Meng $\mathrm{Hu}^{3, \mathrm{~d}}$ \\ ${ }^{1}$ Hainan University, State Key Laboratory of Marine Resource Utilization in South China Sea, Haikou, Hainan, 570228, China \\ ${ }^{1}$ Suzhou North America High School, Suzhou Jiangsu China \\ ${ }^{2}$ Hainan University, State Key Laboratory of Marine Resource Utilization in South China Sea, Haikou, Hainan, 570228, China \\ ${ }^{3}$ International Youth Talent Exchange Center
}

\begin{abstract}
This study is based on our iGEM (international genetically engineered machine) 2019 competition project in which an in vitro model was established to simulate the human monitoring and regulation of blood glucose level using the "liver-on-a-chip" and a genetically engineered bacterium capable of producing proinsulin efficiently. The microfluidic device is able to accommodate cellular chassis loaded with biological parts for diabetic treatment. In addition, electrochemical biosensors were designed to detect the differential glucose concentration from the both chambers of the organ chip. The model can test different chemicals and organs, when the components in the channels and cells are altered. We have thus accomplished an in vitro model of how the proinsulin generated by engineered bacteria works on liver cells. In the near future, our research paradigm will be shifted to bacterial implantation in the human intestines to replace pancreas for the automatic secretion of insulin for diabetic patients.
\end{abstract}

\section{Introduction}

A World Health Organization revealed that there were already 422 million diabetics in 2014 and the number continued to rise each year. If we are to fight against diabetes, we need to first understand its mechanism. Healthy people use their pancreas to secret enough insulin for effective cellular glucose consumption, so their blood sugar level remains low. Diabetic patients can neither produce insulin by their pancreas at all, nor produce it enough, so that their glucose in blood cannot be used by human body. As a result, their blood glucose maintains at high level, eventually leading to health complications such poor blood circulations, damaged vision and nerves, kidney failure, cardiovascular diseases, even heat attacks and stroke [1]. There are two types of diabetes: Type 1 and Type 2. Type 1 diabetes results from autoimmunemediated destruction of the pancreatic $\beta$-cell, resulting in the need for exogenous insulin treatment. Type 2 diabetes is often a milder form of diabetes than type 1. With Type 2 diabetes, the pancreas usually produces some insulin. But either the amount of insulin produced is not enough for the body's needs, or the body's cells are resistant to it. There is no cure for diabetes so far, but we can control its additional harm to organs and nerves. Early phases of type 2 diabetes can be treated with dietary control and regular exercise; however, the treatment to type 1 diabetes is dependent on insulin injection which requires patients to monitor their blood sugar level and inject insulin once or several times a day [2].
In human body, proinsulin is synthesized in pancreatic $\beta$-cells to enable the downstream insulin secretion into blood. When the blood glucose concentration increases, insulin synthesis accelerates. At the same time, a small portion of proinsulin without protease hydrolysis enters the blood circulation. As the only blood glucose reducer, insulin operates mainly through the regulation of hepatic metabolism for the glucose uptake by lever cells [3]. When the level of glycogen reduces and the concentration of insulin rises, liver cells start to increase the uptake of glucose, synthesis of glycogen, suppression of hepatic glucose production (HGP), and the induction of the synthesis of fatty acids for energy storage and further utilization [4]. We can assume proinsulin to have similar functions but is one tenth as affective as insulin.

Previous research has shown that intravenous injections of proinsulin in animal or human result in a decrease in blood glucose. This suggests that proinsulin has similar functions of lowering blood glucose level as insulin. Proinsulin is a precursor to insulin biosynthesis which can stimulate livers to convert glucose to glycogen through binding to insulin receptor in the liver membrane.

What if diabetes could be treated only with implantation of bacteria in our guts to replace painful daily insulin injection? Nowadays the technique of insulin synthesis through cultivation of prokaryotic cells has been well developed. It has already formed a whole industry for insulin production. However, we found that a complicated protein, like insulin, is hard to be synthesized by a bacterium due to the difficulty of cleaving off the $\mathrm{C}$ peptide chain in proinsulin [5]. Researchers from the 
National University of Australia showed a promising future for the implementation of insulin in 2017 [6]. There is not enough convincing experimental data for its clinical application. We thus decided to construct our own biological parts around proinsulin synthesis for this project.

In this work, we planned to construct a "smart" bacterium with the ability to monitor the blood sugar concentration, conduct signal transduction, and implement synthesis and secretion of active insulin. The genetically engineered microbes were implanted within the human gastrointestinal tract to replace/compensate the function of pancreas for insulin secretion. Synthetic biology involved modification of living systems with engineering formality in the way that biological parts were constructed, and assembled as device. The device was then integrated into the molecular chassis to create novel function/products. Our project aimed at applications on human body in the future, we have made an in vitro model on the liver-on-a-chip, which could be used to simulate the human organ environment.

There is an overview of our model below in Fig.1.

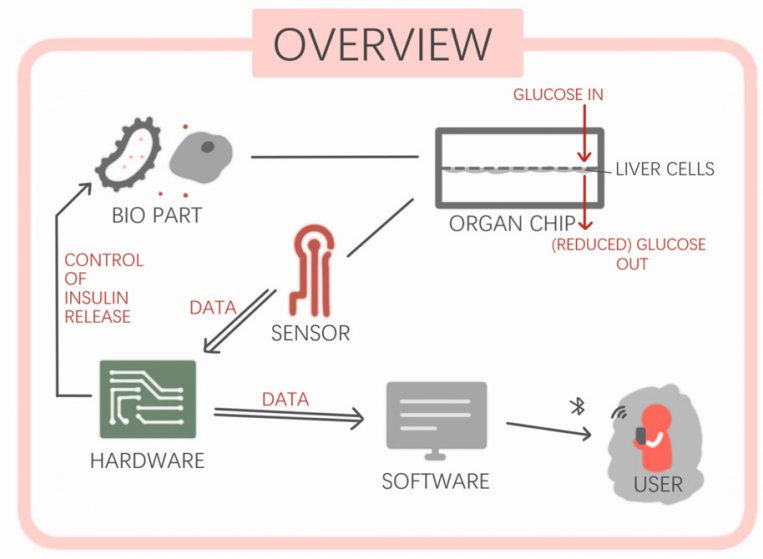

Figure 1: The Overview of The Test.

\section{Methods and Materials}

\subsection{Strains and Culture Conditions}

Bacillus subtilis is a rod-shaped, Gram-positive soil bacterium. We used it as the chassis for transformation of proinsulin genes. Standard LB formulation $(10 \mathrm{~g} \mathrm{NaCl}, 10$ $\mathrm{g}$ tryptone, $5 \mathrm{~g}$ yeast extract per liter, $\mathrm{pH} 7$ ) was used to culture Bacillus subtilis in liquid media.

Hepatocytes are specialized epithelial cells as the main cell type of the liver cell lines, hepatocytes, or more specifically human hepatocarcinoma cell line 2, with the growth medium: DMEM $+10 \%$ serum were transplanted onto the microfluidic device to grow for the construction of "Liver-on-a-chip".

\subsection{Genes and Transformation Vectors}

\subsubsection{HPl gene.}

As the starting step, human proinsulin coding gene (HPI) was synthesized with the sequence of HPI gene as follows:

GAAGAAGCTGAAGCTAAGAGAATGGCGCTTT GGATGAGACTGCTGCCGCTGCTGGCACTTCTTG CGCTTTGGGGACCGGACCCAGCAGCGGCATTTG TTAATCAACATCTTTGTGGCTCACATCTTGTGGA AGCACTTTATCTTGTTTGCGGAGAAAGAGGATT TTTCTATACACCGAAAACAAGAAGAGAAGCAGA AGATCTTCAGGTTGGACAGGTTGAACTGGGCGG CGGACCGGGCGCAGGATCACTTCAGCCGCTGGC ACTGGAAGGCTCACTGCAAAAAAGAGGCATTGT GGAACAATGCTGTACAAGCATTTGCAGCCTTTA TCAGCTGGAAAATTATTGCAATTAA

\subsubsection{Spacer Peptide}

In order to obtain higher proinsulin secretion, we have introduced a spacer peptide between the secretory signal peptides and proinsulin. Nucleotide sequence of spacer peptide: EEAEAKR

Final sequence for spacer peptide and proinsulin: GAAGAAGCTGAAGCTAAGAGA. Through experiments it is shown that the insert of our spacer peptide can notably increase the production of proinsulin.

\subsubsection{Vector}

Human proinsulin gene (HPI) was constructed on the ends of BamHI and EcoRI of the pHY-P43 transformation vector, the recombinant plasmid of pHY-P43-HPI was obtained.

\subsubsection{P43 Promoter}

Promoter P43 is a well characterized, strong promoter of Bacillus subtilis expression system. It is derived from cytidine deaminase (cdd) gene in B. subtilis and is often used to express different target proteins [7]. We used it together with HPI to form the biological part. According to previous experiments, P43 showed the highest expression capability amongst PHpaII, PBcaprE, PluxS, PgsiB, PyxiE, and itself [8]. In our own characterization experiment, we tested P43 against other common promoters, GroE, SecA, YxiE, and Ylbp. Nonetheless, P43 came on top in terms of expression quantities. It was evident that the usage of P43 ensured the proinsulin to be expressed to the utmost extent.

\subsubsection{Colony PCR.}

Colony PCR was implemented to confirm successful transformation of HPI gene into the host E. coli cells. Electrophoresis image demonstrated the presence of a gene of length $250 \sim 500 \mathrm{bp}$, which is the proinsulin gene, in the E. coli constructs. To ensure that the gene between length 250 500 bp was HPI instead of some other genes, the band was extracted from the gel and sequenced. The results show that it matches the sequence of the HPI genes we originally used for synthesis. 


\subsubsection{Gene Transformation}

Specific primers were synthesized to ligate HPI gene into the plasmid pHY-P43. The gene in this plasmid is of the length of $345 \mathrm{bp}$ after successful ligation. Genetically engineered E. coli strains bearing HPI gene were cultured overnight. The recombinant plasmid was extracted from E. coli and transformed into B. subtilis WB800N. We presumed that the modified $\mathrm{B}$. subtilis is able to produce proinsulin. Three different validations were performed.

\subsection{Protein Electrophoresis}

\subsubsection{Plasmid Construction}

After the HPI gene was inserted into pHY-P43 plasmid, the length of the plasmid with HPI gene was found to be $5.5 \mathrm{~K} \mathrm{bp}$. The electrophoresis image confirmed that the recombinant plasmid was constructed successfully.

\subsection{Sensors}

The electrochemical biosensor is a device that can transform chemical quantities (contained by organic matters including human body) into electric quantities (sensible and "understandable" by electronic devices) for signal detection. We chose this kind of sensor because of its fast measurements, sensitivity and ability to perform measurements on complicated solutions (turbid/opaque) [9]. A Typical blood sugar electrochemical sensor contained three electrodes: working electrode (the electrode in an electrochemical system on which the reaction of interest is occurring), counter electrode (an electrode which is used to close the current circuit in the electrochemical cell) and reference electrode (an electrode which has a stable and well-known electrode potential and it was used as a point of reference in the electrochemical cell for the potential control and measurement).

Correlation between glucose concentration and current was then made. 12 readings were taken for 12 different standard solutions. We applied linear regression to the data and made the line of best-fit which is $\mathrm{I}=75.43 \mathrm{c}+1.31$. The correlation coefficient is 0.976 . This means that the equation constructed should be very close to the actual value according to mathematical calculations. Also, it indicted that the sensors were precise.

\subsection{Microfluidic device for construction of liver- on-a-Chip}

Since the optimal adhesion of mammalian cells is critical in determining the cell viability and proliferation on substrate surface, we need to increase adhesion and viability. The PET membrane of the microfluidic device was specially treated as follows. Membrane was subjected to oxygen plasma for $3 \mathrm{~min}$ in the plasma cleaner, followed by immersing them in $10 \%$ APTES (3Aminopropyltriethoxysilane) at $50{ }^{\circ} \mathrm{C}$ for $2 \mathrm{~h}$. The
APTES solution was removed, and the samples were washed twice in nuclease-free water. The PET membrane was further immersed in a $2.5 \%$ GA (Glutaraldehyde) solution at room temperature for $1 \mathrm{~h}$. GA was then removed, and the samples were washed twice in nucleasefree water. Then, the PET membrane was immersed in either $0.1 \mathrm{mg} / \mathrm{mL}$ of a collagen type 1 (Col1) solution and stored at $4{ }^{\circ} \mathrm{C}$ overnight. Finally, the protein solution was removed, and the samples were washed twice with PBS (Phosphate buffer saline). The PET membrane was sterilized under UV light for $60 \mathrm{~min}$ prior to cell culture experiments [10].

We designed the liver-on-a-chip model with SolidWorks software. The microfluidics model composed of a liver-on-a-chip, dual channel electrochemical sensors, home-made data acquisition board, and our biological parts. If B. subtilis produced sufficient proinsulin, the liver cells grown on the organ chip should absorb glucose, and lower down the glucose concentration. As a result, the sensors should detect a decrease in current, which is directly proportional to glucose level. More details can be seen in the following organ-on-a-chip section.

\subsection{Heading Level 2}

\subsubsection{Hardware}

The hardware was home-made. The electrical circuit for our detection system adopts modular design that monitors the reactions on two electrochemical sensors and collects data from them. Functional modules include double threeelectrode electrochemical sensor module, signal acquisition and coordination module, data processing module, wireless communication module, and power module.

\subsubsection{Dual Three-Electrode Electrochemical Sensor} Module. This sensor module transduces the concentrations of the glucose in the liver-on-chip. The difference in the data collected by two sensors (one in engineering bacteria medium and the other in liver cell medium) indicates the effectiveness of the proinsulin that was produced by the engineering bacteria that we built in this project.

\subsubsection{Signal Acquisition and Coordination Module.} This module is composed of a front-end amplifier with current voltage converter and a band-pass filter. MAX9913 is a rail-to-rail amplifier for the three-electrode sensor designed by Maxim, which integrates two operational amplifiers $\mathrm{A}$ and $\mathrm{B}$. The non-inverting input of Amplifier A connects to the DAC chip, inverting input connects to the reference electrode, and the output connects to the counter electrode. The non-inverting input of Amplifier B connects to the DAC chip, and its inverting input connects to the working electrode. When the circuit is functioning, DAC releases constant voltage $\mathrm{Vmd}$ and Vmd+Vbias. By virtual short concept, the voltage at reference electrode is clamped to Vmd, the voltage at working electrode is clamped to Vmd+Vbias, and the 
voltage at counter electrode is 0 . By virtual open concept, current signals from working electrode are converted to voltage signals through a $25 \mathrm{k} \Omega$ resistor where the output voltage Vout $=\mathrm{Vmd}+\mathrm{Vbias}+\mathrm{R} * \mathrm{i}$. The band-pass filter applies the Butterworth filter design, composed of a second-order low-pass filter and a second-order high-pass filter. The bandwidth of our filter is from $0.05 \mathrm{~Hz}$ to $5 \mathrm{~Hz}$. The Gain Bandwidth Product of our design is approximately 25 , calculated by multiplying the amplifier's bandwidth and the gain at which the bandwidth is measured, which indicates that the accuracy of our design is not affected. The Butterworth filter design is advantageous for its easy-to-operate frame and functionality. Controlled by an analog switch, amplified signals pass through the filter. Then, the purified signals are transformed into digital signals by an ADC (Analogto-Digital Converter) and sent to data processing module.

2.6.1.3 Data Processing Module. Microcontroller NUC120LD2BN is adopted as the core controller in our system for its advantages: high speed, low energy consumption, and strong immunity to interference. NUC120LD2BN embeds Cortex ${ }^{\mathrm{TM}_{-} \mathrm{M} 0}$ core running up to $50 \mathrm{MHz}$ with $64 \mathrm{~K}$-byte embedded flash, 8K-byte embedded SRAM, and 4K-byte loader ROM for the ISP. It also embeds 8x12-bit ADCs, 2 UARTs (UniversalAsynchronous-Receiver/Transmitter), 4x32-bit timers, and 4 PMWs (Pulse-Width Modulation). The functions of NUC120LD2BN can satisfy the requirement of our project, and the low energy consumption accords with the portable device. The build-in ADC of NUC120 transforms analog signals into digital signals. Then, the data are calculated and sent through UART to wireless communication module that transfers data to the terminal.

2.6.1.4 Wireless Communication Module. RF-BM-ND01, which is based on Bluetooth 4.0 with the features of low power consumption, small size, long transmission distance, strong anti-jamming capability, and etc., is adopted as the wireless communication module for this project. Bluetooth is a wireless technology for shortdistance data communication with low energy consumption. The strong compatibility of Bluetooth enables any user with a smart phone to interact with our device. The inherent security of Bluetooth in data communication also protects privacy of our users by preventing information leakage.

\subsubsection{Software}

The main goal of this app is to demonstrate the current blood sugar level and the historical data. It also helps track the records of drug releasing. The connection between the device and the app is based on Bluetooth 4.0. Then the data are demonstrated in a chart form, which enables users to easily understand their health conditions. The chart could alter among three different scales - hours, days, and months. Users can input an exact time to search for the blood sugar level at that time. Once users register their own accounts, they can set personal information - such as avatars, age, and type of diabetes - and access historical information on their mobile phones.

Bluetooth: In Bluetooth section, we set our app as the central and the device as the peripherals. First, we import the database CoreBluetooth and define several variables to store the data. Then, we determine the Bluetooth status of the phone and find the equipment that meets the requirements. Finally, the connection event is triggered by clicking the corresponding cell.

SwiftChart is introduced as a database. Because our data are illustrated in three-time scales - day, month and year - three arrays are used to store data in different tables. Refer to the function in the database for drawing graphs.

\section{Results and Discussions}

\subsection{Biological Design}

Our biological goal was to construct a genetically engineered bacterium capable of producing proinsulin efficiently. Our target gene ( $354 \mathrm{bp}$ ) has been modified to obtain higher expression and compatibility. Through the modification to the N-terminal sequence, the chassis obtained a more efficient gene expression; through codon optimization, the sequence of HPI gene is RFC 10 compatible.

\subsubsection{Result of analysis to restriction site.}

Screened with 117 enzymes, 9 sites found

AlwNI CAGNNN/CTG 1: 237

ApaBI GCANNNNN/TGC 1: 41

BglII A/GATCT 1: 177

Bsp1407I T/GTACA 1: 286

CspI CG/GWCCG 1: 211

Eco57I CTGAAGNNNNNNNNNNNNNNNN/ 2: 166,214

PvuII CAG/CTG 1: 312

XmnI GAANN/NNTTC 1: 179

The gene in this plasmid is of the length of $345 \mathrm{bp}$ after successful ligation, as shown in the gel electrophoresis image (Fig. 2). Fig.2 shows the results of protein electrophoresis to both unmodified bacteria and the bacteria that contain recombinant DNA. It indicates that bacteria with original HPI gene secreted a small amount of proinsulin that is not enough to be perceived by naked eyes. 


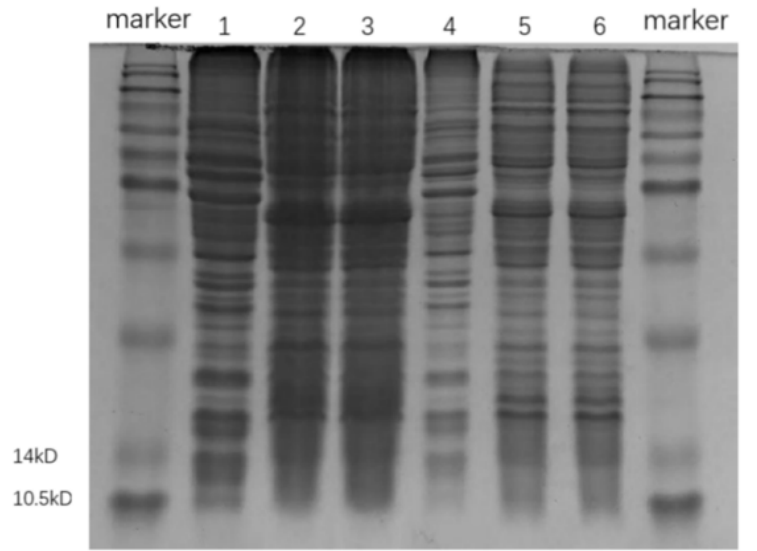

A)

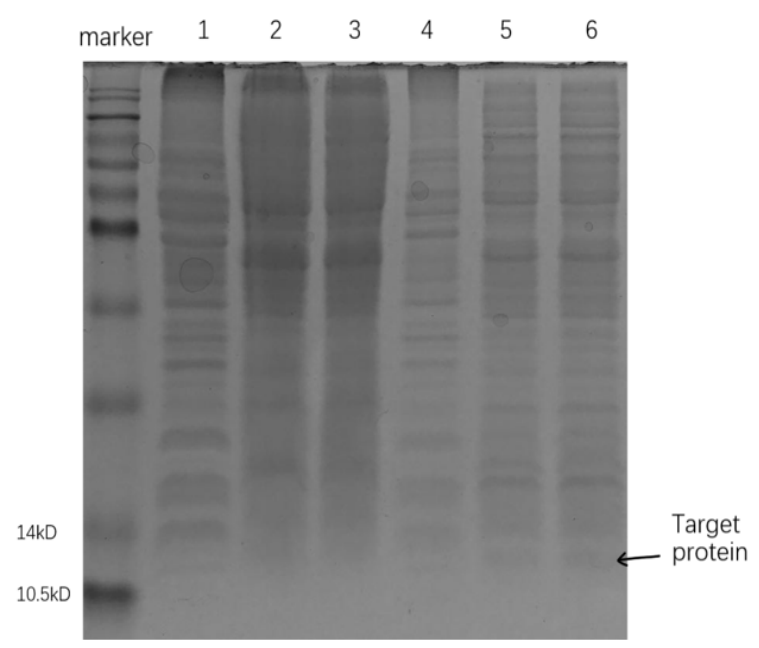

B)

Figure 2: A) The SDS-PAGE Result of Dissolved Bacteria with Unmodified HPI Gene(Bba_K1328003). Lane 1 Is Negative Control--The Supernatant Remained after the Collection of WB800N. Lane 2-3 Are Supernatant of Sample 1 And 2. LANE 4-6 Are 10-Times-Diluted Supertanants of Lane $1-3$.

B) The SDS-PAGE Result Of Bacteria with Codon-Optimized and N-Terminal-Modified HPI Gene. Lane 1 is Negative Control--The Supernatant Remained after the Collection of WB800N. Lane 2-3 are Supernatant of Sample 13 And 16.

Lane 4-6 are 5-Times-Diluted Supertanants of Lane 1-3.

The target protein bands exist in expected size, where proinsulin is about to show--at $10.5 \mathrm{kD}$ to $14 \mathrm{kD}$. Bacteria with modified HPI gene produce large amount of target protein. The modification to HPI sequence has successfully increases the production of protein.

\subsubsection{Protein Electrophoresis}

Insert paragraph text here. Insert paragraph text here. Insert paragraph text here. Insert paragraph text here. Insert paragraph text here. Insert paragraph text here. Insert paragraph text here. Insert paragraph text here. Insert paragraph text here. Insert paragraph text here. Insert paragraph text here.

B. subtilis was cultured for two days. Then all the proteins from the bacteria were extracted to test for proinsulin by protein electrophoresis. Human proinsulin has a molecular weight of about $12 \mathrm{kDa}$, so there should be an additional band between 14 and $10.5 \mathrm{kDa}$ on the protein electrophoresis image, comparing to the control group that did not contain any HPI genes. Despite numerous attempts, we couldn't obtain a clear picture showing that particular band.

In Fig.3A, 1 is the control group where the bacteria don't contain HPI gene. 2 and 3 are the experimental group where the bacteria contain HPI gene. 4,5 , and 6 are $10 x$ dilutions of 1,2 , and 3 respectively. There is no visible difference between the bands of the control group and experimental group, meaning that the engineered bacteria didn't produce HPI as we have expected.

After rigorously analyzing our previous experiments for any mistakes, we found that the inability to produce the band on the gel image was because low expression of proinsulin. Although a strong promoter was used, the quantity of proinsulin obtained was still low. It is evident that the expression efficiency has to be increased. After a careful consideration, we decided to add a spacer peptide on the upstream region of the HPI gene. The results indicate a visible additional band between $14 \mathrm{kDa}$ and $10.5 \mathrm{kDa}$, comparing to the control group ( 1 and 4 on Fig. $6)$. It is seen that the spacer peptide has actually enhance the gene expression, and the bacteria synthesized our desired protein, proinsulin.

In Fig. $3 \mathrm{~B}, 1$ is the control group where the bacteria don't contain HPI gene. 2 and 3 are the experimental group where the bacteria contain HPI gene. 4,5 , and 6 are 10x dilutions of 1, 2, and 3 respectively. There are bands of 5 and 6 with deep color between $14 \mathrm{kDa}$ band (grey) and $10.5 \mathrm{kDa}$ band (dark grey) of marker which 4 lacks. This shows that the bacteria do produce proinsulin.

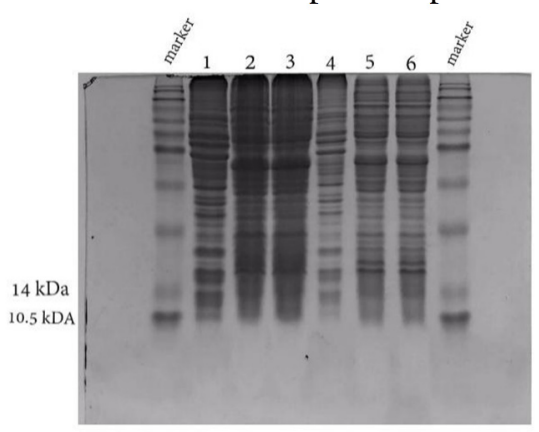

A)

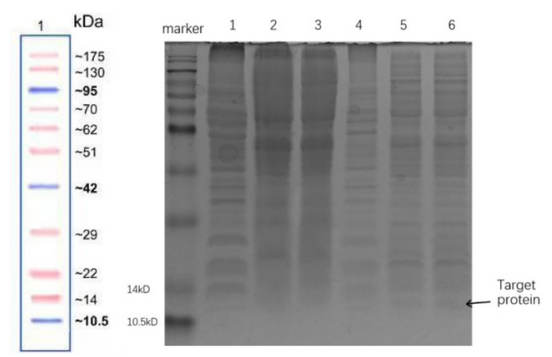

B)

Figure 3: A) Results from Protein Electrophoresis before Spacer Peptide is Added. B) Protein Electrophoresis after Spacer Peptide is Added. 


\subsubsection{Construction of Chassis}

Bacillus subtilis is able to form a receptive state under natural conditions, to accept foreign genes, and thus to provide a platform as host bacteria for expression [11]. However, Bacillus subtilis itself may produce a large number of proteases that degrade foreign proteins, especially the most widely used strain B. subtilis 168 , which is able to secrete a large number of proteases, causing great inconvenience for the expression of foreign proteases [12]. For strain B. subtilis 168,8 proteaseinactivated strains B. subtilis WB800 were obtained by mutation inactivation method. In addition, WB800N possessed resistance against neomycin.

B. Subtilis WB800N was modified to synthesize proinsulin and store them in cytoplasm. After transformation, they were transplanted onto the top chamber of our liver-on-a-chip as a proinsulin source for liver cells. Liver cells were cultivated in the bottom chamber of the chip and attached to the PET membrane, which has small pores that only allows exchange of small molecules. When proinsulin was released, it was able to go through the pores and promote the liver cell to synthesize glycogen from glucose in the lumen, achieving the reduction of glucose concentration inside the bottom chamber.

\subsection{Temperature Negative Feedback Control of Glucose}

In order to enable an "efficient system" for rapid release of proinsulin to regulate glucose level, an extracellular lysozyme was used. Lysozyme can destroy peptidoglycan, an important component in bacteria's cell wall, to release proinsulin in the fastest way.

In the real world, excessive amount of insulin in the blood stream may cause hypoglycemia which could be fatal for diabetic patients. To make our in vitro model as realistic as possible, we set out to control the amount of proinsulin released to prevent the glucose concentration from both increasing or decreasing to a dangerous level $(>180 \mathrm{mg} / \mathrm{dL}$ or $<70 \mathrm{mg} / \mathrm{dL})$ [13]. There already exists a negative feedback loop by insulin-glycogen binding in our body which has inspired us to construct a similar loop to achieve the same effect [14].

Synthetic biology allows the existence of nonbiological parts. Temperature in the bacterial chamber of the liver-on-a-chip was closely monitored and adjusted as a control mechanism. When the blood glucose sensor detected a rise in glucose level, the temperature would be increased to the optimum temperature for effective digestion effect of lysozymes. Adversely, when the glucose level started to drop, the temperature would be reduced to lower down the activity of lysozyme. It would thus manipulate the amount of proinsulin. Fig. 4 elaborates the mechanism of this negative feedback loop.

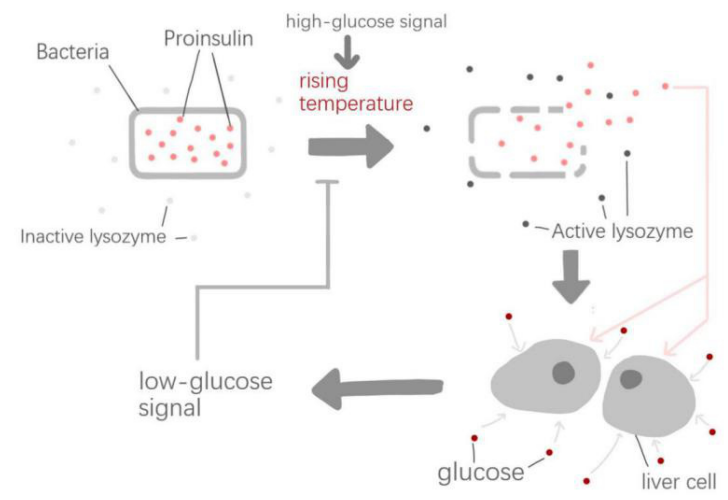

Figure 4: A Negative Feed-Back Loop, Based on the Control of Temperature and the Release of Lysozyme.

By using B. subtilis WB800N as the cellular chassis, active proinsulin was synthesized efficiently. The mutant could be applied to our in vitro blood glucose regulation model, and when the specific B. subtilis chassis was loaded with the heterologous modules, novel diabetic treatment is achievable.

\subsection{Sensor}

\subsubsection{Design}

Of many applications of electrochemical sensors, the most common one would be blood sugar monitoring. In order to monitor blood glucose concentration, enzymatic electrochemistry was applied. In an enzyme-based electrochemical sensor, enzymes served as catalysts in the reduction reaction caused by variation in glucose concentration and finally produced an electrical current as sensor outputs. The redox reaction is as followed [15]:

$$
\begin{aligned}
& \text { Glucose }+\mathrm{O}_{2} \stackrel{\mathrm{GO}_{\mathrm{x}}}{\Rightarrow} \text { Gluconic acid }+\mathrm{H}_{2} \mathrm{O}_{2} \\
& \text { Glucose }+\mathrm{GOx}_{(\mathrm{ox})} \rightarrow \text { Gluconic acid }+\mathrm{GO}_{\mathrm{x}_{(\mathrm{red})}} \\
& \mathrm{GOX}_{(\mathrm{red})}+2 \mathrm{M}_{(\mathrm{ox})} \rightarrow \mathrm{GOx}_{(\mathrm{ox})}+2 \mathrm{M}_{(\mathrm{red})}+2 \mathrm{H}^{+} \\
& 2 \mathrm{M}_{(\mathrm{red})} \rightarrow 2 \mathrm{M}_{(\mathrm{ox})}+2 \mathrm{e}^{-}
\end{aligned}
$$

We have designed our own enzyme-based electrochemical sensors to evaluate the change of glucose concentration in our project since commercial electrochemical sensors have short life span, and their enzymes on the working electrodes are easy to fall off. To improve our sensor's usage, we applied an entrapping method for the immobilization of enzymes, such as glucose oxidase on the electrode. The entrapping method possessed a comparably high rate of enzyme recovery which made it the best method for glucose monitoring.

Our sensor generally contained three layers. The PET layer at the bottom served as carrier of the components; the paste of silver chloride layer in the middle served as conductor of electricity; the carbon paste layer at the top served as both the working electrode and the counter 
electrode. The design of the electrochemical sensor is shown in Fig. 5. COSMOL Multiphysics was used to simulate the blood glucose regulation.

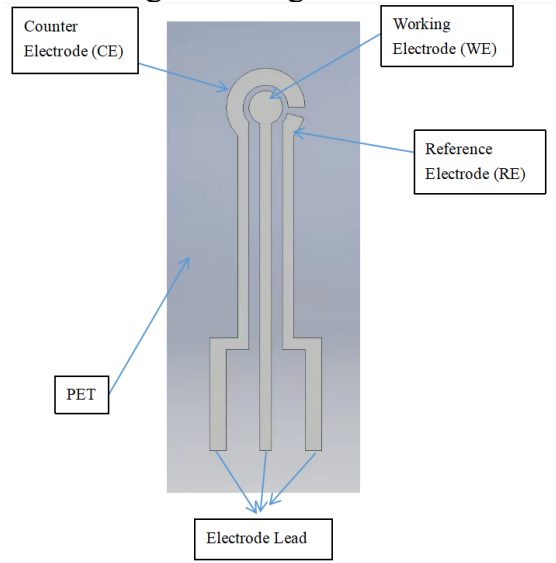

Figure 5: The Design of the Electrochemical Sensor.

Firstly, we had the PET layer $(27 \mathrm{~cm} \times 36 \mathrm{~cm})$ as the basis. Secondly, we used silk-screen printing (printing plate $32 \mathrm{~cm} \times 44 \mathrm{~cm})$ to prepare the first layer of the electrodes (Working electrode, Counter electrode) with carbon paste (material). After sintering $\left(90^{\circ} \mathrm{C}\right.$ in sinter box for 10 minutes), the second layer of the electrodes were printed with Ag (material) and further sintered. On the top of the second layer of WE, we prepared the layer of enzymes (which was glucose oxidase (GOx) of 10 $\mathrm{mg} / \mathrm{mL}$ in $1 \mathrm{x}$ PBS solution) and applied the protection (the entrapping method) on the layer. The Gox solution would be the mixture of $0.5 \mathrm{~mL}$ of $1 \mathrm{M}$ acetic acid and 4 ug of Chitosan.

\subsubsection{Testing}

After function of the electrodes was confirmed, testing on the sensor was done to demonstrate its overall operationality. The sensor was inserted into a glucose solution of concentration $0.1 \mathrm{mM} / \mathrm{L}$. After an interval of $50 \mathrm{~s}, 5 \mu \mathrm{L}$ of glucose is added into the solution. The sensor measured the current output. Fig. 36 showed a clear trend between current and time. As time increases, more glucose was added into the solution, so the current increased, from $0 \mu \mathrm{A}$ to $195 \mu \mathrm{A}$. Thus, the sensors have the capability to detect glucose concentration in the whole range since there is clearly a relationship between the glucose concentration and the current measured by the sensor.

CV (Cyclic Voltammetry) curve in electrochemical workstation was generated and tested for the performance and practicability of our sensors. As a type of electrochenmical measurement, it measured the peak current (and the corresponding bias voltage) and the reversibility of the redox reaction by manipulating the electrode potential. A CV curve would be the graph of the current versus potential. According to the CV graph shown in Fig. 6, the bias voltage should be approximately $0.15 \mathrm{~V}$.

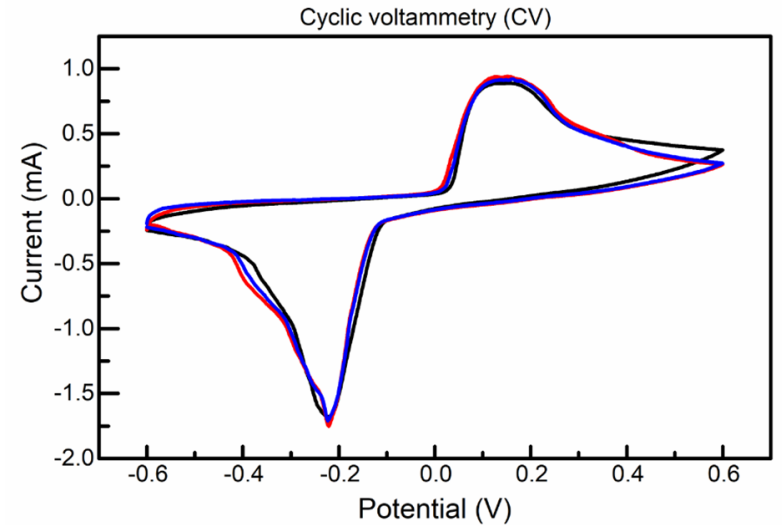

Figure 6: CV Curve.

\subsection{Liver-on-a-chip}

In recent years, organs-on-a-chips have garnered increasing attention due to both ethical and scientific reasons. An extensive interest has been shown by many pharmaceutical, food and cosmetic industries in applying these organ-on-a-chip for study of drug, nutrient and xenobiotic absorption and possible toxic effects [16]. The field of organs-on-a-chip is based on technological advances in tissue engineering and microfluidics as well as insights into the extraction, culture and maturation of human cells, enabling the design of customized cellular microenvironments with precise fluidic, mechanical and structural control [17]. So, the organ chip could serve as in alternation of animal and human cells/organs in the biology study, especially when it comes to drug invention.

3.4.1 Liver Structure and Liver-on-a-chip. Human liver is consisted of hepatocyte cells. There are around 2.5 billion Hepatocytes in the liver. Hepatocytes are polygonal, about $20-30 \mu \mathrm{m}$ in diameter. In consideration of the structure of human liver in vivo, we designed ourmicrofluidic device based on the minimum functional unit of the liver, the operation principle was shown in Fig. 7.

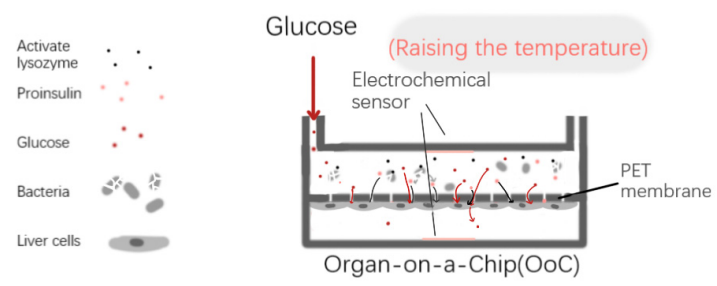

Figure 7: The Schematic Diagram of the Liver-On-A-Chip

\subsubsection{Design of liver-on-a-chip}

We built a microenvironment in the liver-on-a-chip to simulate the fast release of proinsulin from $B$. subtilis WB800N to stimulate Hepatocytes to uptake glucose when the blood glucose level was elevated. The microfluidic device contained two channels, two sensors and some layers to fix the functional parts. 
For the microfluidic device, PDMS (Polydimethylsiloxane) was used. PDMS has shown to possess numerous properties that offer advantages over many other available biomaterials such as silicon, bioglass, and other polymers. Furthermore, PDMS can be easily tuned by the base/curing agent's ratio to cover a wide range of physiologically relevant elastic modulus for mechanobiological studies as compared to other materials used for similar purposes, such as polyacrylamide gels, poly (ethylene glycol), and hyaluronan. In addition, PDMS is a preferred material for cell-based platforms used in biomedical devices and fundamental studies due to its optical transparency, gas permeability, nontoxicity, and cost effectiveness [10]. A porous PET membrane (0.4 $\mu \mathrm{m}$ pores, $10 \mu \mathrm{m}$ thick) was sandwiched between the two microfluidic channels to separate the two channels, cells attached with a growing on the PET membrane as well. For the diameter of the porous, nanoscale control over pore diameter offers the advantage of modulating cell communication routes while maintaining and even improving permeability when incorporated within ultrathin membranes [18].

\subsubsection{Dual Sensor}

In order to detect the differential glucose concentrations after proinsulin was released, we designed dual electrochemical sensors, one on the top of the liver-on-achip and the other at the bottom of the organ chip.

The top channel of the design is used to mimic the "blood" of human. The sensor in the top part of the liveron-a-chip contacts with the solution in the top channel to detect the concentration of the glucose in "blood". Moreover, the bacterial solution was added into the top channel to mimic the injection of proinsulin.

The sensor in the bottom part contacted with the solution in the bottom channel. During this process, the liver cell line was cultured and used to mimic the liver side of the body for monitoring the concentration of the glucose after the effects of liver with proinsulin.

\subsubsection{Cell cultures in the liver-on-a-chip}

Human hepatocarcinoma cell line 2 was cultured at $37^{\circ} \mathrm{C}$ and $5 \% \mathrm{CO} 2$ with the growth medium:DMEM $+10 \%$ serum. When the liver cell cultures reached a density of 2 x 105 cells per $\mathrm{mL}$, they were inoculated in the bottom channel of the liver-on-a-chip. Then the organ chip was put upside down in the incubator at $37^{\circ} \mathrm{C}$ for $24 \mathrm{~h}$ to allow hepatocyte attachment on the porous membrane. After that, the chip was turned into the right side, the culture solutions were changed every 12 hours. After $28 \mathrm{~h}$, we infuse our engineering bacteria solution into the top channel of the chip. The closed loop glucose concentration control microfluidic chip has thus been prepared.

3.4.5 Fluorescence intensity. DAPI is a fluorescent dye that binds strongly to DNA. The fluorescence intensity of
DAPI molecules bound to double-stranded DNA is increased by about 20 times, which is commonly observed with fluorescence microscopy. According to the fluorescence intensity, the amount of DNA can be determined. In addition, because DAPI can penetrate intact cell membranes, it can be used to stain living and fixed cells. Due to these properties of DAPI, we use it as our fluorescent dye and help us identify the living Hepatocyte cells in the chip. Besides DAPI, we also use CDFDA, which can combine with active protoplast. Therefore, we can identify live cells by using CDFDA. The result is shown in Fig.8, DAPI in blue and CDFDA in red. This picture shows that our cells have already clung to the chip and begin to grow.

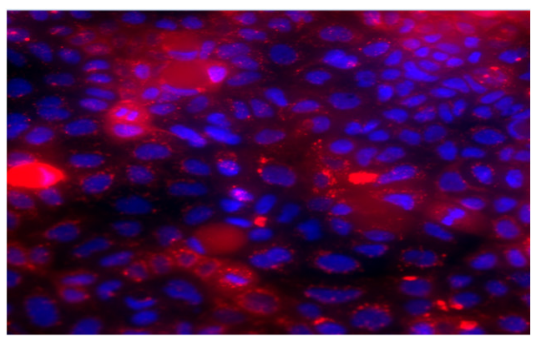

Figure 8: Staining Result (Blue: DAPI, Red: CDFDA)

Then different concentrations of glucose were loaded into the chip to test whether the liver-on-a-chip worked correctly with electrochemical sensors real-time monitoring. After the liver-on-a-chip was fabricated, the sensors were assembled into the chip. One sensor on the top part to monitor the concentration of the glucose in the "blood" side, another sensor on the bottom to monitor the glucose in the "liver" side. After the chip assembled, liver cell suspension solution was loaded into the chip by pipette. Moreover, the pipette with liver cell culture medium was kept inserting in the chip to provide the nutrition for the cells. Then put the chip into an incubator for culturing.

All the experiments with liver done in the incubator (the readout device was not in the incubator, which was connected with chip by wire), the picture in below shown the connected chip with the readout setup. Due to all the experiments were in the incubator, this picture shown the testing without any cells.

\subsection{Model}

This module addressed two significant aspects in our design - electrochemical simulation and mathematical modeling.

\subsubsection{Electrochemical Simulation}

The electrochemical sensor was simulated by COMSUL Multiphysics software. We adopted the multi-physics coupling simulation, and adjusted the external glucose concentration to $0.005 \mathrm{~mol} / \mathrm{m} 3$, the concentration of ferrocyanide to $0.001 \mathrm{~mol} / \mathrm{m} 3$, and the concentration of ferricyanide to $50 \mathrm{~mol} / \mathrm{m} 3$. The maximum reaction rate 
was $0.015 \mathrm{~mol} /(\mathrm{m} 3 * \mathrm{~s})$. Perform a software simulation to obtain the calibration curve for glucose.

\subsubsection{Mathematical Model}

Our mathematical modeling tries to describe performance of the electrochemical sensor in response to the glucose concentrations. The aim for mathematical modeling is to establish the relationship between the Differential equations are introduced to describe the relation of blood glucose concentration and temperature that determines the proinsulin production.

To test the performance of the sensors, electric potential was altered between the range $-0.6 \mathrm{~V}$ to $0.6 \mathrm{~V}$ on the working electrode of the sensors. A current was produced against the reference electrode which was $0 \mathrm{~V}$ all the time. The current was measured continuously, and the electric potential against current was plotted below in Fig. 35. The change of current, $0.8 \mathrm{~mA}$ to $-1.8 \mathrm{~mA}$, on the graph with respect to electric potential shows that the electrodes on the sensors were functional, and the medium on the sensors were conductive.

\subsection{Glucose regulation}

To confirm that it's accurate to deduce the unknown glucose concentration just from the current data by using the equation, $\mathrm{c}=(\mathrm{I}-1.31) / 75.43$, four tests of known concentration of glucose solution $(0.2 \mathrm{mmol} / \mathrm{L}, 0.4$ $\mathrm{mmol} / \mathrm{L}, 0.8 \mathrm{mmol} / \mathrm{L}, 1.6 \mathrm{mmol} / \mathrm{L})$ were implemented.

In order to make sure that two glucose concentration sensors can work side by side, two sensors were dipped into two solutions (a $2 \mathrm{mM}$ glucose solution and a 1x PBS solution). The current outputs from the two sensors were different. The reading for the sensor to the glucose solution was in the range of $3838 \sim 3508 \mathrm{mV}$, while the other one was in the range of $2732 \sim 2683 \mathrm{mV}$. The difference indicates that our sensors are capable of working together at the same time and still obtaining accurate values.

\subsubsection{Fabrication of liver-on-a-chip}

The organ chip was fabricated and incorporated with the functional biochemical sensors. Several tests on the chip were carried out before the bacteria was injected. First, diffusion rate of glucose inside the organ on chip was tested. The two chambers were filled with PBS. Glucose was injected into the upper chamber (simulating the blood stream). After the current readings of the top and bottom chamber stabilized, another batch of glucose was added. The two sensors on the upper and lower chamber continuously recorded current. From the graph (Fig. 9A), it is seen that after 40-80 seconds, the glucose concentration became relatively equal in the two chambers. It can be inferred that little time was taken for glucose to achieve equilibrium between the top and bottom chamber, and glucose can diffuse very easily in between.

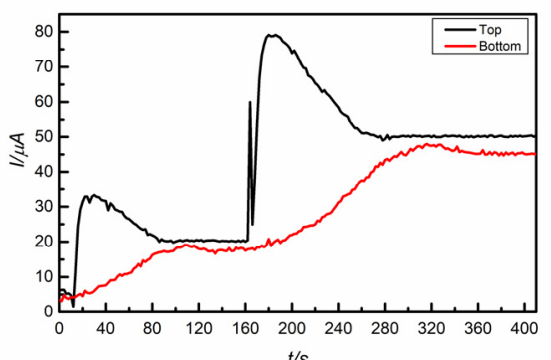

A)

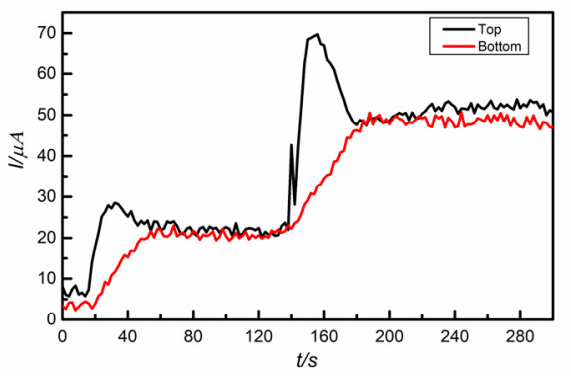

B)

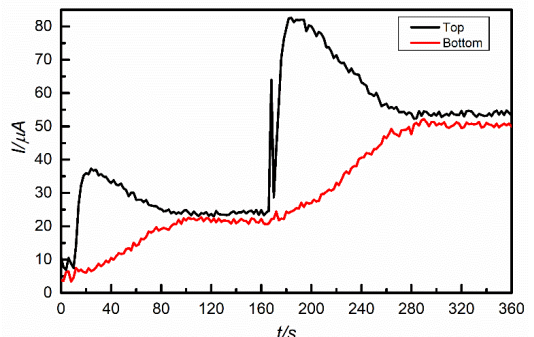

C)

Figure 9: The Current Measurements of The Top and Bottom Chamber A) Before and after Glucose Addition only into The Upper Chamber; B) Containing Liver Cells before and after Glucose Addition; C) Containing Bacteria, and the Bottom Chamber Containing Liver Cells Before and after Glucose Addition.

In order to mimic the liver function, cancerous liver cell lines, hepatocytes, were transplanted onto the lower chamber of our organ chip. Two fluorescent dyes, DAPI and CDFDA, were used to assess the liver cell growth. After 48 hours, several images (Fig. 8) were taken using fluorescence microscope. The abundance of blue-colored nucleus, stained by DAPI, in these pictures confirm that there were plenty of viable liver cells present in the chip. Here, the red color in the surroundings signifies MRP2 activity for the hepatocyte efflux transporter. This means that liver cells are functioning normally.

With the assurance of liver cells growing finely, the same test of glucose diffusion was carried out again. It took much longer time for the stabilization of glucose concentration in the lower chamber, on average 80 seconds. We concluded that the liver cell impeded the diffusion of glucose, thus the time for equilibrium increased (Fig. 9B).

In our last experiment, proinsulin-bearing B. subtilis cells were injected into the organ chip. After 48 hours, lysozyme was added to release the proinsulin. The recorded time for glucose equilibrium was about 90 seconds (Fig. 9C), longer than previous experiment 
without proinsulin-producing B. subtilis. Also, the glucose concentration (current) for the bottom chamber was significantly less than that of the top chamber. The above results provide convincing evidence that the release of proinsulin triggered the liver cells to take in glucose. The glucose level was lowered down within our in vitro model by the proinsulin from engineered $\mathrm{B}$. subtilis, the objective of our project to simulate bacterial supply of insulin in vitro for the treatment of diabetes is achieved.

\section{Conclusions}

We have successfully mimicked the human liver in absorbing glucose in an in vitro environment. In order to achieve it, Unique combination of electrochemical sensors, liver-in-a-chip and use of lysozymes for fast insulin release was designed in this work. The in vitro model in this study for blood glucose monitoring and regulation is a crucial first step towards our ultimate goal for bacterial implantation in the human intestines to replace pancreas for the automatic secretion of insulin for diabetic patients.

We are striving to develop a new method to release insulin in our body instead of the traditional painful way that would be a remarkable breakthrough, even though there is still a large gap to close. We have to figure out how to establish a co-culture system that can function as both a glucose detector and a medicine producer, how to conduct the implantation of such a bacterial system into a patient's body, and whether the bacteria would exert potential harm to the patients or the environment.

\section{ACKNOWLEDGMENTS}

Special thanks to Wei Wei who offer us tremendous support with access to the laboratories at Hainan University and offer the idea of project. Special thanks to Hainan University offering all the material, labor, technology, and all the equipment that our team needs to finish the project. Finally, great thanks to our team members: Mingyuan Li, Kehan Zhan, Shunzhi Wen, Can Li, Keyi Huang, Qingyue Gao, En Lei, Hanwen Wang, Yarong Li and Siyi Tang.

\section{REFERENCES}

1. P Hossain, B Kawar and ME Nahas (2007). Perspective: Obesity and Diabetes in the Developing World - A Growing Challenge. The New England Journal of Medicine, 356: 213-215

2. M Massi-Benedetti (2003). Glimerpiride in type 2 diabetes mellitus: A review of the worldwide therapeutic experience. Clinical Therapeutics, 25(3): 799-816

3. Paul M. Titchenell, et al (2017). Unraveling the regulation of Hepatic Metabolism by Insulin. Trends Endocrinol Metab, 28(7): 497-505
4. LinHV, et al (2011). Hormonal Regulation of Hepatic glucose Production in Health and Disease. Cell Metab, 14: 9-19

5. X Wang (2012). Research Progress and Prospect of Bacillus subtilis. Journal of the Graduates Sun YatSen University (Natural Sciences 、 Medicine), 10(003): 14-23

6. C.R. Kahn \& M.F. White (1988). The Insulin recepter and the molecular mechanism of insulin action. J Clin Invest, 82(4): 1151-1156

7. Kitabchi, A. E. (1977). Proinsulin and C-peptide: A review. Metabolism, 26(5), 547-587. doi:10.1016/0026-0495(77)90099-3

8. Spizizen J (1958). Transformation of biochemically deficient strains of Bacillus Subtilis by deoxyribonucleate. Proceedings of the National Academy of Sciences of the United States of America, 44(10): 1072-1078.

9. IDC Technologies. Types of Biosensors, www.idconline.com/technical_references/pdfs/chemical_engi neering/Type s_of_biosensors.pdf.

10. Kuddannaya, S., et al. (2013). Surface chemical modification of poly(dimethylsiloxane) for the enhanced adhesion and proliferation of mesenchymal stem cells. ACS Appl Mater Interfaces, 5(19): 977784.

11. LÜ Dan, YAN Yali, JING Lifang, ZHANG Qiurong, CHANG Li, DIAO Aipo, LI Yuyin (2017). Soluble Expression of Recombinant Human Proinsulin in E.coli. Journal of Tianjin University of Science\&Technology, 32(5): 1-5.

12. Song W, Nie Y, Mu X Q, et al (2016). Enhancement of extracellular expression of Bacillus naganoensis pullulanase from recombinant Bacillus subtilis: Effects of promoter and host. Protein Expr Purif, 124: 23-31.

13. Vavrová L, Muchová K, Barák I (2010). Comparison of different Bacillus subtilis expression systems. Research in Microbiology, 161(9): 791-797.

14. Checking Your Blood Glucose. American Diabetes Association. Updated Oct 09, http://www.diabetes.org/living-withdiabetes/treatment-and-care/blood-glucosecontrol/checking-your-blood-glucose.html

15. Sensor - an Overview | ScienceDirect Topics, www.sciencedirect.com/topics/engineering/glucosesensor.

16. Srinivasan, B., et al. (2015). TEER measurement techniques for in vitro barrier model systems. J Lab Autom, 20(2): 107-26.

17. Zhang, B., et al. (2018). Advances in organ-on-a-chip engineering. Nature Reviews Materials, 3(8): 257278.

18. Chung, H.H., et al. (2018). Use of porous membranes in tissue barrier and co-culture models. Lab Chip, 18(12): 1671-1689. 
19. Song, Yafeng et al (2016). "Promoter Screening from Bacillus subtilis in Various Conditions Hunting for Synthetic Biology and Industrial Applications." PloS one, 11(7): e0158447.

doi: 10.1371/journal.pone.0158447

20. Heyoung Jeong, et al (2018). "Complete Genome Sequence of Bacillus subtilis Strain WB800N, an extracellular Protease-Deficient Derivative of Strain 168." Microbiol Resour Announc, 7(18): e01380-18

21. Grieshaber, Dorothee, et al (2008). "Electrochemical Biosensors - Sensor Principles and Architectures." Sensors (Basel, Switzerland), Molecular Diversity Preservation International (MDPI), 7 Mar. 2008, www.ncbi.nlm.nih.gov/pmc/articles/PMC3663003/. 